\section{Tumores do Córtex Adrenal na Infância}

\section{RESUMO}

O tumor do córtex adrenal (TCA) na infância é raro. Entretanto, no Paraná sua incidência é 15 vezes maior do que a observada em outros países. Descrevemos as características clínicas, laboratoriais, tratamento e evolução de 125 pacientes atendidos em uma única instituição no Paraná. A média de idade ao diagnóstico foi de 4,3 anos, com uma relação feminino:masculino de 2,6:1. As formas clínicas mais comuns foram virilização isolada $(51,2 \%)$ e virilização e síndrome de Cushing (42\%). Em 4,8\% dos casos, os tumores foram não-funcionantes. Dois pacientes $(1,6 \%)$ apresentaram síndrome de Cushing isolado e $1(0,8 \%)$ síndrome de Conn. Hipertensão arterial ocorreu em $56 \%$ dos pacientes. O único tratamento curativo é a cirurgia. Os dados da nossa casuística mostram que estádio clínico 1 do TCA, ausência de trombo intravenoso e ausência de spillage durante a cirurgia estão associados a uma maior sobrevida. (Arq Bras Endocrinol Metab 2004;48/5:651658)

Descritores: Carcinoma; Córtex adrenal; Infância

\begin{abstract}
Childhood Adrenocortical Tumors.

Adrenocortical tumors (ACT) in children are uncommon. However, the incidence of these tumors in Paraná, Brazil, is 15 times higher than that worldwide. We describe the clinical, laboratory and treatment characteristics and outcome of 125 patients treated in a single institution in the State of Paraná. The median age at diagnosis was 4.3 years, with a female:male ratio of 2.6:1. The most common forms of presentation were isolated virilization (51.2\%) and virilization and Cushing's syndrome (42\%). Nonfunctioning tumors comprised $4.8 \%$ of the cases. Two patients ( $1.6 \%)$ had isolated Cushing's syndrome and 1 ( $0.8 \%)$ had Conn's syndrome. Fifty-six percent presented hypertension. Surgery is the only curative treatment. Our data show that disease stage 1, absence of spillage during surgery and absence of intravenous thrombus were associated with better survival rates. (Arq Bras Endocrinol Metab 2004;48/5:651658)
\end{abstract}

Keywords: Carcinoma; Adrenal cortical; Childhood

$\mathbf{O}$ TUMOR DO CÓRTEX ADRENAL (TCA) pode ocorrer em qualquer idade. Uma distribuição bimodal tem sido relatada, ocorrendo um primeiro pico antes dos 5 anos de idade e um segundo entre a $4^{\text {a }}$ e $5^{\text {a }}$ décadas de vida (1). O TCA é raro na infância. Nos Estados Unidos, a incidência anual estimada é de 0,3 casos/1.000.000 em indivíduos abaixo de 15 anos de idade (2), sendo diagnosticados 25 casos novos/ano (3). Entretanto, nas regiões Sul e Sudeste do Brasil, em especial nos estados do Paraná e São Paulo, esta incidência está aumentada em 10 a 15 vezes, chegando a 4,2

\section{artigo original}

\author{
Rosana Marques Pereiva \\ Edson Michalkiewicz \\ Fabiano Sandrini \\ Bonald C. Figueivedo \\ Mara Pianovski \\ Susana Nesi França \\ Margaret C.S. Boguszewski \\ Orival Costa \\ Izrail Cat \\ Luiz de Lacerda Filho \\ Romolo Sandrini
}

Departamento de Pediatria, Hospital de Clínicas, Universidade Federal do Paraná

(UFPR),

Unidade de Endocrinologia

Pediátrica (RMP, FS, SNF,

MCSB, LLF, RS), Serviço de

Hemato-Oncologia Pediátrica

(MP), Unidade de Terapia

Intensiva (OC,IC), Centro de

Genética Molecular e Pesquisa em

Câncer na Infáncia (BCF) e

Serviço de Cirurgia Oncológica

Pediátrica, Hospital Erasto

Gaertner (EM), Curitiba, PR.

Recebido em 10/06/04 Aceito em 18/06/04 


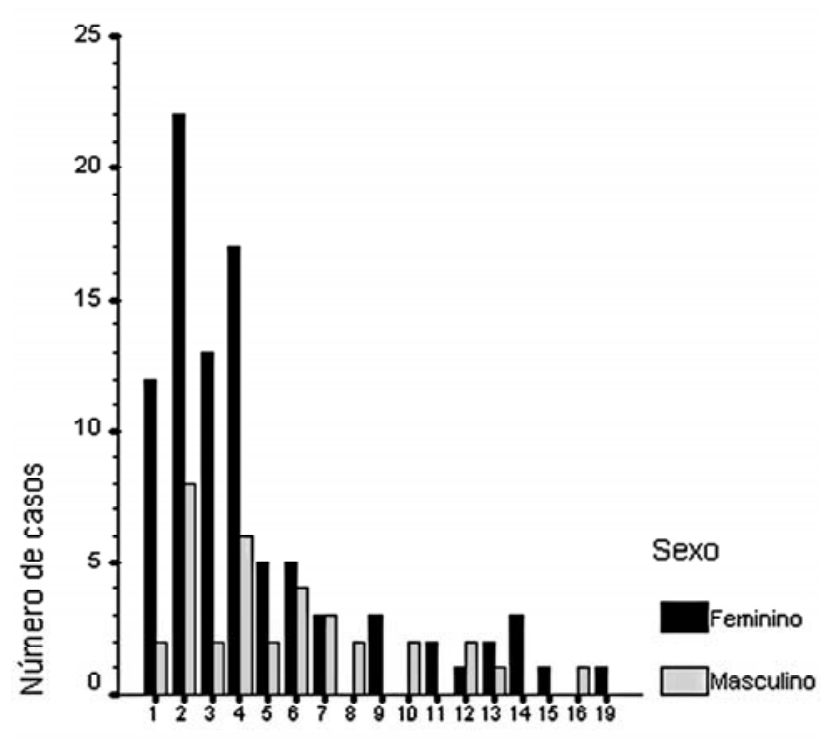

Idade (anos)

Figura 1. Distribuição dos 125 pacientes com TCA de acordo com idade e sexo.

casos/ 1.000.000 (4). Na Unidade de Endocrinologia Pediátrica (UEP) do Hospital de Clínicas da Universidade Federal do Paraná são diagnosticados 6 a 8 casos novos/ano; entre 1966 e 2003 foram atendidas 125 crianças portadoras de TCA.

$\mathrm{Na}$ literatura mundial, descreve-se a relação entre o TCA e a síndrome de Beckwith-Wiedemann e a síndrome de Li-Fraumeni (5). Estudos realizados no Brasil não demonstraram que a maior incidência do TCA seja devida a estas associações $(1,4)$.

Em 2001 foi identificada, nos pacientes atendidos no Paraná, a mutação germinativa R337H no gene supressor tumoral $\mathrm{p} 53$, presente em 35 de 36 pacientes em acompanhamento na UEP (6). Posteriormente, a mesma mutação foi descrita em 19 pacientes de São Paulo, sendo 5 adultos (7). De 11 pacientes avaliados no St. Jude Children's Research Hospital, 5 apresentaram mutação do p53, mas nenhum a mutação descrita no Brasil (8).

Um achado não descrito anteriormente, a presença de mais de 1 caso de TCA em uma mesma família, passou a ser observado (9). Atualmente, são acompanhadas na UEP 8 famílias com 2 ou mais casos de TCA na infância.

Identificou-se, também, que a mutação germinativa $\mathrm{R} 337 \mathrm{H}$ do gene p53 está presente em familiares sadios dos pacientes portadores de TCA. De 65 pacientes e 698 familiares do lado do progenitor com a mutação, avaliados na UEP, 61 pacientes $(93,8 \%)$ e 274 familiares $(39,2 \%)$ apresen-

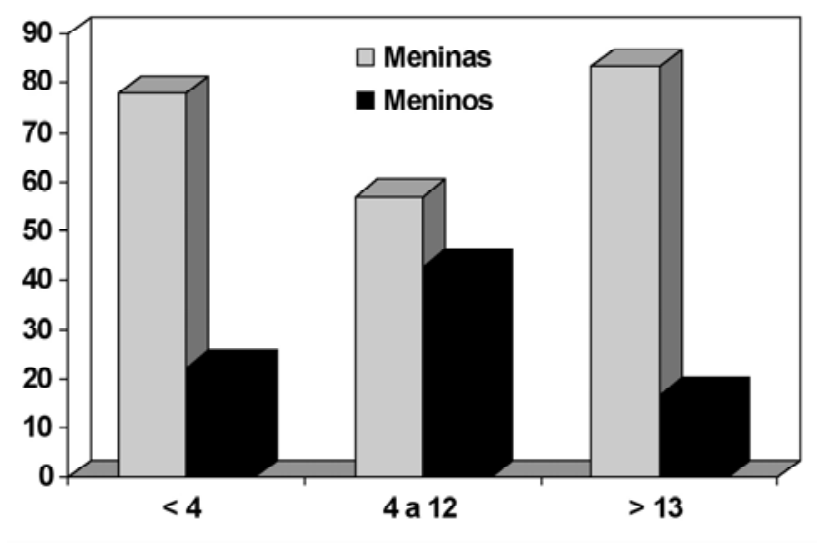

Figura 2. Distribuição percentual dos pacientes com TCA de acordo com sexo e grupo etário.

taram a mutação; destes, $61(22,3 \%)$ são crianças com menos de 15 anos de idade. Nenhum dos 232 indivíduos do lado do progenitor não afetado apresentou a mutação (Figueiredo e cols., submetido para publicação).

A penetrância estimada de TCA entre os indivíduos menores de 15 anos de idade portadores da mutação é de 10,4\%. Entretanto, quando há mais de 1 caso de TCA na família, a penetrância é de 14,1\% (Figueiredo e cols., submetido para publicação). Este dado nos motivou a fazer acompanhamento médico das crianças sem tumor, portadoras da mutação R337H p53.

\section{ACHADOS CLÍNICOS E LABORATORIAIS}

Apesar de diferenças geográficas quanto à incidência do TCA na infância, as características clínicas do tumor, nas casuísticas americanas e européias, são semelhantes àquelas dos pacientes avaliados em Curitiba. De 42 crianças com TCA tratadas em centros franceses durante um período de 22 anos, a média de idade ao diagnóstico foi de 3,9 anos, houve freqüência maior no sexo feminino e $90 \%$ dos pacientes apresentavam virilização (10).

Dos 125 pacientes atendidos na UEP, a média de idade ao diagnóstico foi de 4,3 anos e mediana de 3,2 anos; $66,4 \%$ dos pacientes tinham entre 0 e 4 anos de idade ( 3 casos de tumores congênitos); $28,7 \%$ entre 4 e 12 anos; $4,9 \%$ tinham mais que 13 anos (figura 1 ). 
Noventa crianças $(72 \%)$ eram meninas e 35 (28\%) meninos com uma relação de 2,6:1. Entretanto, a relação entre os sexos mostrou um padrão diferente quando avaliada por faixa etária. Nas crianças com menos de quatro anos de idade, a relação feminino: masculino foi de 5,3:1; entre 4 e 12 anos 1,3:1 e nos adolescentes ( $\geq 13$ anos) 5:1 (figura 2 ).

A média do intervalo de tempo entre o início dos sintomas e o diagnóstico foi de 11,13 meses, com uma mediana de 6 meses, embora nos últimos anos haja uma tendência à diminuição deste intervalo.

As manifestações clínicas mais freqüentes do TCA estão relacionadas ao aumento da secreção de hormônios pelo córtex adrenal. Raramente estes tumores são não-funcionantes. As formas de apresentação clínica são a virilização, a síndrome de Cushing e a hipertensão mineralocorticóide. São sinais comuns pubarca, hipertrofia do clitóris ou pênis, hirsutismo, acne, aumento da massa muscular, aumento da velocidade de crescimento, irritabilidade, ganho de peso, alteração do timbre da voz, hipertensão arterial, convulsões, entre outros. Estrias estiveram presentes em apenas 2 pacientes.

Com muita freqüência o tumor é palpável $(4,11)$. A presença de circulação colateral abdominal sugere obstrução da veia cava pela presença de trombo venoso ou compressão (10).

Em 51,2\% dos pacientes, o quadro clínico foi de virilização isolada; em $42 \%$, a virilização estava associada a sinais e sintomas da síndrome de Cushing (síndrome mista). Em 2 pacientes (1,6\%) ocorreu a síndrome de Cushing isolado e 1 paciente $(0,8 \%)$ apresentou síndrome de Conn. Os tumores não-funcionantes corresponderam a $4,8 \%$ dos casos.

Hipertensão arterial foi observada, por ocasião do diagnóstico, em $56 \%$ dos pacientes e esteve presente em todas as formas clínicas do TCA. Em 9 pacientes, a hipertensão foi severa a ponto de causar encefalopatia hipertensiva com crise convulsiva. A hipertensão arterial é atribuída, em parte, à produção aumentada de glicocorticóides (na maioria dos casos) e/ou mineralocorticóides pelo tumor. Contudo, 13,6\% dos pacientes com quadro clínico e laboratorial de virilização isolada também apresentaram hipertensão, cuja patogênese pode estar relacionada à compressão da artéria renal pelo tumor (8) ou à secreção de um ou mais esteróides adrenais que não foram mensurados (12). Não é infreqüente a hipertensão arterial persistir por dias ou semanas após a ressecção do tumor (dados não publicados). Na nossa experiência no manejo da hipertensão, em geral há uma boa resposta à terapêutica com inibidores da ECA, utilizados como monoterapia ou em associação com diurético, e por vezes com cetoconazol nas formas severas.

Nenhum paciente apresentou tumor bilateral, e as glândulas direita e esquerda foram afetadas em proporções semelhantes. Uma paciente com a mutação R337H p53 apresentou um tumor pequeno do lado esquerdo, que foi retirado aos 4 anos de idade; 11 anos após retornou com um tumor na glândula direita, metástases pulmonares e trombo na veia cava que se estendia até o átrio direito. Embora seja um caso isolado, a presença da mutação indica a necessidade de acompanhamento permanente dos pacientes.

O diagnóstico do TCA é baseado nos achados clínicos, nas dosagens hormonais e nos exames de imagem. A presença de sinais e sintomas decorrentes da produção aumentada de 2 ou mais hormônios adrenais é altamente sugestiva da presença de TCA. Nas regiões Sul e Sudeste do Brasil, devido à alta incidência do TCA, ao avaliarmos uma criança menor que 6 anos com pubarca, a hipótese de TCA deve ser sempre considerada e investigada. A elevação acentuada dos níveis de sulfato de de-hidroepiandrosterona (S-DHEA) é um achado laboratorial sugestivo da presença do TCA. Na pubarca precoce idiopática, o SDHEA pode estar em níveis normais altos ou discretamente aumentados. Em geral, há também alteração dos níveis de testosterona, 17-hidroxi-progesterona e androstenediona. As dosagens de cortisol plasmático, incluindo-se a avaliação do ritmo circadiano e o teste de supressão com dexametasona, do cortisol urinário livre, da aldosterona plasmática e atividade de renina do plasma são importantes para o diagnóstico e uma avaliação da funcionalidade do tumor.

Os métodos de imagem utilizados para confirmação e localização do tumor e presença ou não de metástases são, preferencialmente, a ressonância nuclear magnética (RNM) e a tomografia axial computadorizada (TAC). A sensibilidade da ecografia para detectar um tumor pequeno está relacionada ao poder de resolução do aparelho utilizado e à experiência do examinador. Em uma avaliação anterior em nosso serviço, a ecografia não detectou a presença do tumor em 3 de 28 pacientes; em todos o tumor foi identificado pela TAC (4). Contudo a ecografia, assim como RNM e a venografia, é útil para identificação de trombos venosos. A RNM é um excelente método para a avaliação do abdome. Entretanto, sua sensibilidade é baixa para a detecção de metástases pulmonares pequenas (13). Utilizamos a TAC como método de imagem de rotina na investigação do 
TCA, por julgarmos ser operacionalmente mais prática a avaliação do paciente com o mesmo exame de imagem para tórax e abdome.

\section{TRATAMENTO}

\section{Cirurgia}

A ressecção completa do tumor e de linfonodos regionais, sem ruptura da cápsula tumoral, é a melhor alternativa terapêutica do TCA. A laparotomia transperitoneal é a técnica mais adequada para ressecção completa do tumor, dos linfonodos retroperitoneais regionais e inventário da cavidade abdominal (14). A ruptura da cápsula do tumor com extravasamento de células neoplásicas no leito cirúrgico ou na cavidade abdominal pode ocorrer antes ou durante a cirurgia e aumenta consideravelmente o risco de recidiva local (8). A ruptura préoperatória é rara (11) e, durante a cirurgia, pode ocorrer devido a dificuldades técnicas para ressecar o tumor, que pode ser extremamente friável e, às vezes, de grande volume.

Cento e vinte e três pacientes foram submetidos à cirurgia curativa ou diagnóstica. A grande maioria dos pacientes $(86,2 \%)$ teve o tumor completamente ressecado; 3,3\% teve ressecção parcial (tumor residual macroscópico) e somente biópsia foi realizada em 10,6\% dos pacientes (na década de 60).

A nosso ver, a experiência atualmente acumulada com a cirurgia laparoscópica retroperitonial não permite sua indicação para retirada de TCA, especialmente pela possibilidade de ruptura de cápsula, recidiva local e disseminação do tumor (15-17).

Trombo tumoral intravenoso foi encontrado em 19,6\% de 92 pacientes investigados, principalmente nas veias cava, renal, supra-renal e intracardíaco. Uma vez diagnosticado o trombo, deve-se ressecá-lo juntamente com o tumor primário. Em doença metastática ao diagnóstico ou em recidiva, local ou à distância, deve-se tentar a ressecção completa seguindo os mesmos princípios para o tumor primário (18). Esta conduta tem curado ou prolongado a sobrevida de alguns pacientes. Um deles está sem a doença há 20 anos, após 2 cirurgias para retirada de recidiva local e 1 para hemi-hepatectomia por metástase hepática. Outra paciente, com diagnóstico aos 9 meses de idade, apresentou 10 recidivas locais e à distância e foi operada em cada recidiva, tendo sobrevivido por cinco anos.

A maioria dos pacientes sem ressecção completa do tumor morre entre 3 e 12 meses.

Nos pacientes que apresentam hipercortisolismo, está indicada a reposição hormonal de glicocorticóide durante e após a cirurgia, para prevenir insuficiência supra-renal aguda.

\section{Quimioterapia}

A quimioterapia tem acrescentado pouco impacto à chance de cura. Contudo, nos casos em que a ressecção completa do tumor não é possível e nos tumores metastáticos, algum tipo de tratamento quimioterápico adjuvante, embora controverso, tem sido indicado $(4,19,20)$.

O esquema ICE (ifosfamida, carboplatina e etoposideo), utilizado para tratar algumas crianças com TCA avançado, mostrou-se ineficaz (21).

Em 1998, Berruti e cols. (22) descreveram 2 pacientes com resposta completa e 13 com resposta parcial, taxa de resposta global de $53,5 \%$ após o tratamento com a combinação de cisplatina, doxorrubicina, etoposideo e mitotano.

A dose do mitotano proposta por Berruti e cols. foi por nós modificada (de $4 \mathrm{~g} /$ dia para $4 \mathrm{~g} / \mathrm{m}^{2} /$ dia). Estamos utilizando este esquema para pacientes em estádio 3 ou 4 e para pacientes que tiveram estádio 1 ou 2 inicialmente e recidivaram.

Para acelerar o depósito de mitotano no tecido adiposo, o mesmo é misturado com triglicerídeos de cadeia média e outros alimentos gordurosos rotineiramente ingeridos na dieta do paciente (leite, chocolate, iogurte). Os efeitos colaterais são intensos e freqüentes, incluindo lesão temporária ou permanente da adrenal contralateral, náuseas, vômitos, diarréia, sintomas neurológicos como letargia, sonolência, mudança no humor e, mais raramente, coma. É freqüente a falta de adesão por intolerância gástrica. Para alguns pacientes, temos indicado o uso de sonda nasoenteral para garantir a ingestão da droga.

O mitotano deve ser monitorizado com determinações séricas freqüentes, para manter o nível terapêutico e não tóxico, situado entre 14 e $20 \mathrm{mcg} / \mathrm{ml}(22-26)$.

Em doses $\leq 3 \mathrm{~g} /$ dia, o mitotano induz à supressão da esteroidogênese adrenal; em doses > $3 \mathrm{~g} /$ dia, é observado um efeito adrenocorticolítico. Por isto, reposição de glicocorticóide e mineralocorticóide deve ser iniciada até duas semanas após o início do mitotano (19).

Devido à interferência do mitotano no metabolismo dos esteróides $(19,27,28)$, as doses de reposição, tanto do glicocorticóide como do mineralocorticóide, devem ser maiores que a fisiológica. Utilizamos preferencialmente prednisona (10 a $\left.15 \mathrm{mg} / \mathrm{m}^{2} / \mathrm{dia}\right)$, dividida em 2 a 3 tomadas ou dexametasona ( 2 a $3 \mathrm{mg} / \mathrm{m}^{2} / \mathrm{dia}$ ) em 2 tomadas; e fluodrocortisona, 0,15 a $0,2 \mathrm{mg} / \mathrm{dia}$. Em situações de 
estresse, a dose do glicocorticóide deverá ser triplicada, retornando-se à dose habitual após a resolução do fator de estresse.

Controlamos as doses de reposição baseados na avaliação clínica cuidadosa e na monitoração dos níveis de sódio, potássio, glicemia e ACTH.

Não se conhece, ainda, a duração ideal do tratamento com mitotano, mas, nos pacientes que estão em remissão, deve ser mantido por um período mínimo de 18 meses. Suspendemos a quimioterapia nos pacientes que apresentam recidiva durante o tratamento.

Utilizamos o esquema de Berruti e cols. (22) modificado, em 8 pacientes. Resultados preliminares demonstram que é possível conseguir remissão completa e prolongada de lesões metastáticas e/ou pulmonares. Cinco pacientes apresentaram remissão completa, por períodos que variaram de 6 a 20 meses.

Pacientes que recidivam após o uso de quimioterapia e cirurgia têm mau prognóstico.

\section{FATORES PROGNÓSTICOS}

O papel do diagnóstico histológico do TCA para determinação do seu prognóstico é bastante controverso (4). Este fato ocorre porque a diferenciação do TCA em benigno ou maligno, unicamente com os achados histopatológicos, é difícil, se não impossível (29). Contudo, nesta casuística de 125 pacientes, os 20 TCA que tiveram laudo anátomo-patológico de adenoma estão vivos e sem sinais de recidiva tumoral. $\mathrm{Na}$ avaliação de alguns pacientes, a citometria de fluxo não teve correlação com o prognóstico (29).

Devido à dificuldade em relacionar os achados anátomo-patológicos ao prognóstico do TCA, características clínicas, laboratoriais, de tratamento e presença ou não de metástases foram avaliadas e relacionadas à evolução dos pacientes.

Em uma primeira avaliação de 40 pacientes da UEP (4), observou-se que, nos pacientes sem metástase ao diagnóstico, as características clínicas rela-

Tabela 1. Estadiamento do TCA em crianças.

\begin{tabular}{l}
\hline Estádio Descrição \\
\hline I Tumor totalmente ressecado; peso do tumor 200g; \\
ausência de metástase. \\
II Tumor totalmente ressecado com margens cirúrgicas \\
negativas; peso do tumor > 200g; ausência de metás- \\
tase. \\
III Tumor residual (micro ou macroscópico) ou ino- \\
perável, sem metástase. \\
IV Metástase à distância no diagnóstico.
\end{tabular}

cionadas a um pior prognóstico foram idade $\geq 3,5$ anos, intervalo entre o início dos sintomas e o diagnóstico $>6$ meses, volume do tumor $\geq 200 \mathrm{~cm}^{3}$, peso do tumor $\geq 80 \mathrm{~g}$ e excreção urinária de $17-\mathrm{OH} \mathrm{CS} \geq$ $4,0 \mathrm{mg} / \mathrm{m}^{2}$. Entretanto, na análise multivariada, somente o tamanho do tumor estava associado à sobrevida. Com base nestes resultados, propôs-se um estadiamento para TCA em crianças (4).

Com o aumento da casuística e novas análises estatísticas, observou-se que o peso poderia ser utilizado no lugar do volume para facilitar a avaliação do estadiamento (tabela 1 ).

Na nossa série, 63 pacientes $(50,4 \%)$ estavam no estádio clínico (EC) $1 ; 40$ (32\%) no EC $2 ; 12(9,6 \%)$ no EC 3 e $10(8 \%)$ no EC 4.

A maioria $(92,6 \%)$ das crianças abaixo de 4 anos apresentou tumores com EC 1 (66,7\%) e EC 2 (25,9\%). Entre 4 e 12 anos, a maioria encontrava-se no EC 2 (51,4\%), com um número grande de crianças com doença avançada (14,3\% no EC 3 e 17,1\% no EC 4 ).

A publicação dos dados do Registro Internacional do TCA na Infância (7), baseada na avaliação de 254 crianças, das quais $43,3 \%$ da UEP e $36 \%$ de outros centros brasileiros, relacionou a idade $<4$ anos, quadro clínico de virilização isolada, ausência de hipertensão arterial e tumor com peso $\leq 200 \mathrm{~g}$, totalmente ressecado e sem metástases, a um bom prognóstico. A ressecção incompleta do tumor, a ruptura da cápsula durante a cirurgia e a presença de trombo intravenoso são fatores de mau prognóstico, mesmo quando combinado à quimioterapia adjuvante $(8,29)$.

Os pacientes com trombo intravenoso apresentaram sobrevida em cinco anos estatisticamente inferior $(30 \%)$ em relação àqueles que não têm trombo (84\%) (figura 3).

A revisão dos aspectos cirúrgicos de nossa casuística mostrou que a ruptura do tumor (spillage) durante a cirurgia aumenta significativamente a incidência de recidiva local (8) e diminui a sobrevida (figura 4).

A presença de síndrome de Cushing não está relacionada a um pior prognóstico (figura 6).

$\mathrm{O}$ estadiamento do TCA na infância mostrou correlação significativa com a sobrevida (figura 5).

\section{EVOLUÇÃO}

A recidiva local e/ou metástase do tumor ocorreu em 19 pacientes $(15,2 \%)$; destes, 5 apresentavam EC 1 . Uma paciente apresentou um tumor contralateral 11 anos após a cirurgia, com a presença de trombo venoso 


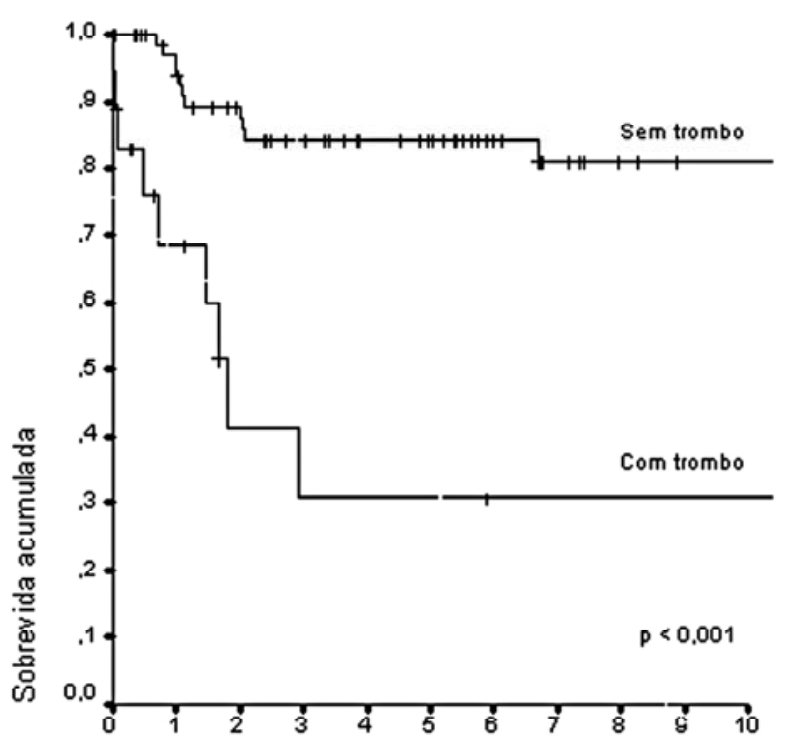

Sobrevida (anos)

Figura 3. Gráfico de Kaplan-Meier, mostrando a sobrevida conforme a presença ou ausência de trombo intravenoso, em 125 pacientes.

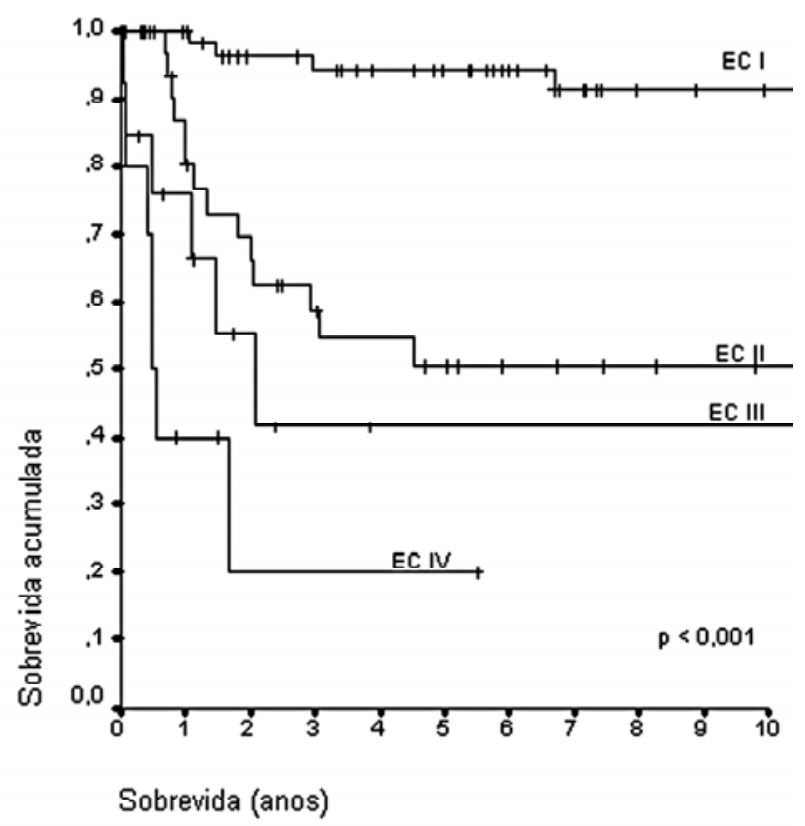

Figura 5. Gráfico de Kaplan-Meier, mostrando a sobrevida conforme o estadiamento clínico, em 125 pacientes. EC: estádio clínico.

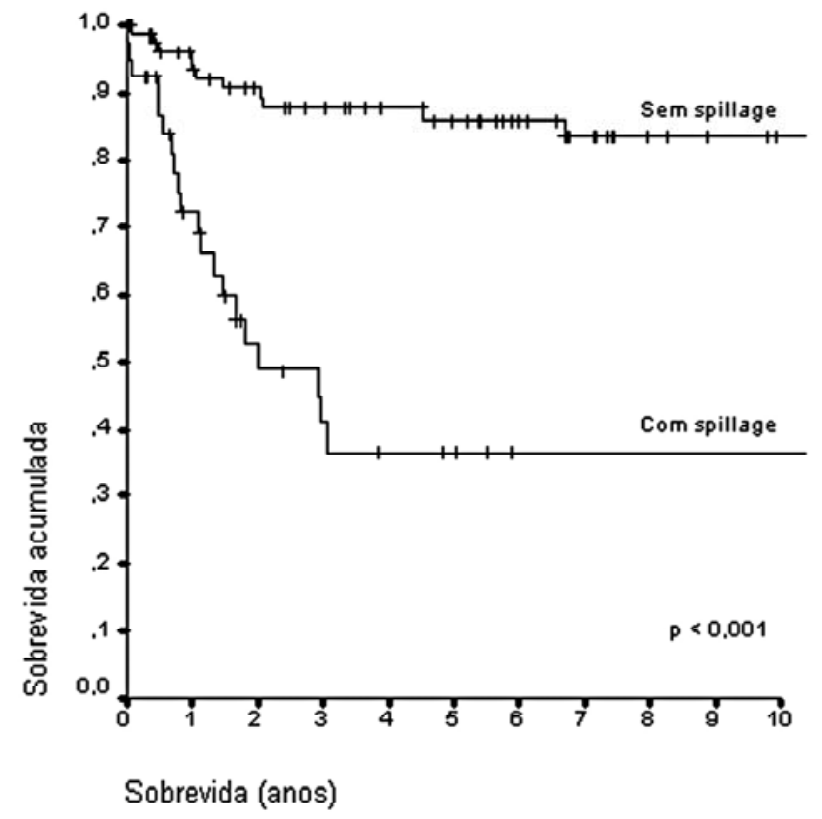

Figura 4. Gráfico de Kaplan-Meier, mostrando a sobrevida conforme a presença ou não de spillage, em 125 pacientes.

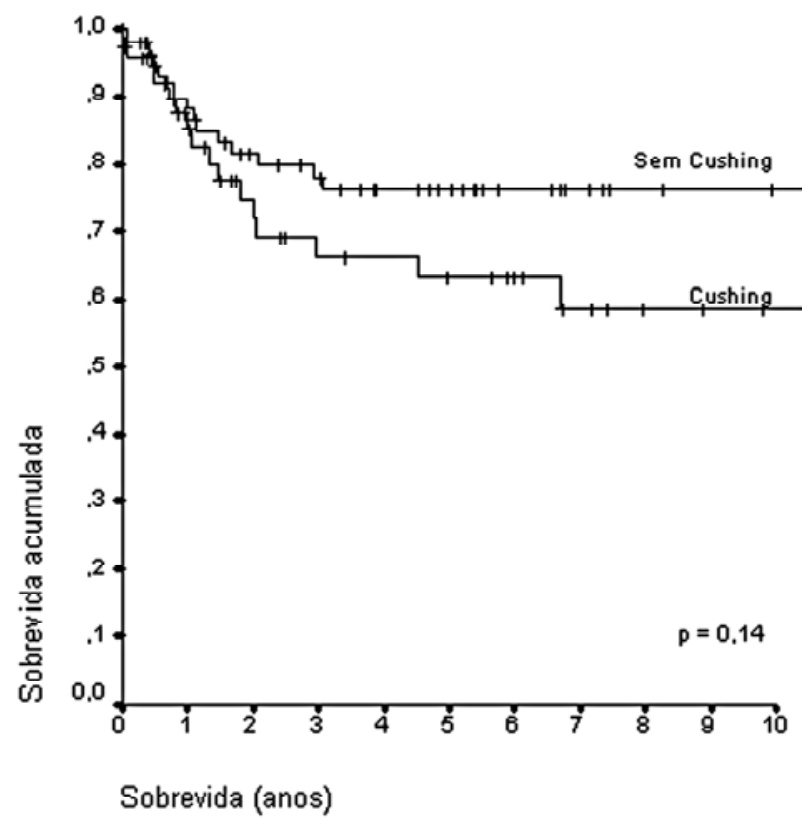

Figura 6. Gráfico de Kaplan-Meier, mostrando a sobrevida conforme a presença de síndrome de Cushing, em 125 pacientes. 
que se estende ao átrio direito.

Oitenta e oito pacientes $(70,4 \%)$ estão vivos e sem sinais de doença atual. Trinta e um pacientes foram a óbito (4 pacientes do EC 1); 5 pacientes com persistência do tumor foram perdidos para o acompanhamento; 1 paciente apresentou recidiva e encontra-se em tratamento.

\section{CONCLUSĀO}

Em resumo, a incidência do TCA no Paraná é 15 vezes maior que a incidência descrita em outros países; a casuística da UEP constitui a maior casuística mundial de TCA na infância de uma única instituição. A presença da mutação germinativa $\mathrm{R} 337 \mathrm{H}$ no gene p53, nos pacientes e familiares sadios, provavelmente está relacionada a este aumento na incidência.

O estadiamento de TCA na infância, a presença de trombo intravenoso, a presença ou não de spillage durante a cirurgia e a retirada completa do tumor e trombos são parâmetros importantes para o prognóstico. Apesar das limitações da quimioterapia no tratamento do TCA, a associação do mitotano ao etoposideo, doxorrubicina e cisplatina tem se mostrado promissora. Contudo, o número de pacientes e o tempo de utilização não permitem conclusões definitivas.

\section{REFERÊNCIAS}

1. Latronico AC, Chrousos GP. Adrenocortical tumors. J Clin Endocrinol Metab 1997;82:1317-24.

2. Young JL, Miller RW. Incidence of malignant tumors in US children. J Pediatr 1975;86:254-8.

3. Wieneck JA, Thompson LDR, Heffess C. Adrenal cortical neoplasm in the pediatric population. Am J Surg Pathol 2003;27:867-81.

4. Sandrini R, Ribeiro RC, DeLacerda L. Childhood adrenocortical tumors. J Clin Endocrinol Metab 1997;82:202731.

5. Koch CA, Pacak K, Chrousos GP. The molecular pathogenesis of hereditary and sporadic adrenocortical and adrenomedullary tumors. J Clin Endocrinol Metab 2002; 87:5367-84.

6. Ribeiro RC, Sandrini F, Figueiredi B, Zambetti GP, Michalkiewicz E, Lafferty AR, et al. An inherited p53 mutation that contributes in a tissue-specific manner to pediatric adrenal cortical carcinoma. Proc Nat Acad Sci USA $2001 ; 98: 9330-5$.

7. Latronico AC, Pinto EM, Domenice S, et al. An inherited mutation outside the highly conserved DNA-binding domain of the p53 tumor suppressor protein in children and adults with sporadic adrenocortical tumors. J Clin Endocrinol Metab 2001;86:4970-3.

8. Michalkiewicz E, Sandrini R, Figueiredo B, et al. Clinical and outcome characteristics of children with adrenocortical tumors: a report from the International Pediatric Adrenocortical Tumor Registry. J Clin Oncol 2004;5:83845.

9. Figueiredo B, Ribeiro R, Ellen M, et al. Multiple childhood adrenocortical tumors in six families from Southern Brazil without Li-Fraumeni syndrome. Horm Res 2000,53 (suppl. 2):15.

10. Lefevre M, Gerard-Marchant R, Gubler JP, Chaussain JL, Lemerle J. Adrenal cortical carcinoma in 42 patients treated from 1958 to 1980 at Villejuif. In: Humphrey GB, Grindey GB Dehner LB et al, editors. Adrenal and endocrine tumors in children. Boston: Martinus Nijhoff Publishers, 1984. p. 256-76.

11. Lack EE, Mulvihill JJ, Travis WD, Kozakewich HOW. Adrenocortical neoplasm in the pediatric and adolescent age group: clinicopathologic study of 30 cases with emphasis on epidemiological and prognostic factors. Pathol Ann 1992;27: 1-53.

12. Kater CE, Biglieri EG, Brust N, et al. Stimulation and suppression of the mineralocorticoid hormones in normal subjects and adrenocortical disorders. Endocr Rev 1989;10:149-64.

13. Kersjes W, Mayer E, Buchenorth M, et al. Diagnosis of pulmonary metastases with turbo-SE MR imaging. Eur Radiol 1997;7:1190-4.

14. Nagesser SK, Kievit J, Hermans J, Krans HM, Van V. The surgical approach to the adrenal gland: a comparison of the retroperitoneal and the transabdominal routes in 326 operations on 284 patients. Jpn J Clin Oncol 2000;30:68-74.

15. Deckers S, Derdelinckx L, Col V, Hamels J, Maiter D. Peritoneal carcinomatosis following laparoscopic resection of an adrenocortical tumor causing primary hyperaldosteronism. Horm Res 1999;52:97-100.

16. Foxius A, Ramboux A, Lefevre Y, Broze B, Hamels J, Squifflet J. Hazards of laparoscopic adrenalectomy for Conn's adenoma. When enthusiasm turns to tragedy. Surg Endosc 1999; 13:715-7.

17. lino K, Oki Y, Sasano H. A case of adrenocortical carcinoma associated with recurrence after laparoscopic surgery. Clin Endocrinol (Oxf) 2000;53:243-8.

18. Pommier RF, Brennan MF. An eleven-year experience with adrenocortical carcinoma. Surgery 1992;112:96370; discussion 970-1.

19. Wajchenberg BL, Pereira MAA, Mendonça BB, et al. Adrenocortical carcinoma. Clinical and laboratory observations. Cancer 2000;88:711-36.

20. Chudler RM, Kay R. Adrenocortical carcinoma in children. Urol Clin North Am 1989;16:469-79.

21. Pianovski MAD, Lacerda Filho L, Figueiredo BC, et al. ICE (Ifosfamide, Carboplatin and Etoposide) combination chemotherapy in the treatment of children with 
advanced adrenocortical carcinoma. Med Ped Oncol 1999:33:279.

22. Berruti A, Terzolo M, Pia A, Angeli A, Dogliotti L, for the Italian Group for the study of adrenal cancer. Mitotane associated with etoposide, doxorrubicin and cisplatin in the treatment of advanced adrenocortical carcinoma. Cancer 1998;83:2194-200.

23. Andersen A, Kasoerkiki-Zaluska AA, Warren DJ. Determination of mitotane (o-p'-DDD) and its metabolites o-p'DDA and o-p'-DDE in plasma by high-performance liquid chromatography. Ther Drug Monit 1999;21:335-40.

24. Andersen A, Warren DJ, Nome O, et al. A high-pressure liquid chromatography method for measuring mitotane (1,1-(o-p'-dichlorodiphenyl)-2-2dichloroethane) and its metabolite 1,1-(o-pl'Dichlorodiphenyl) -2-2-dichloroethene in plasma. Ther Drug Monit 1995;17:526-31.

25. Baudin E; Pellegriti G; Bonnay M, et al. Impact of monitoring plasma 1,1-dichlorodiphenilcichloroethane (0p'DDD) levels on the treatment of patients with adrenocortical carcinoma. Cancer 2001;92:1385-92.
26. Haak HR, Hermans J, van de Velde CJH, et al. Optimal treatment of adrenocortical carcinoma with mitotane: results in a consecutive series of 96 patients. $\mathbf{B r} \mathbf{~ J ~ C a n - ~}$ cer 1994;69:947-51.

27. Hogan TF, Citrin DL, Johnson BM, et al. o,p'-DDD (mitotane) therapy of adrenal cortical carcinoma: observations on drug dosage, toxicity, and steroid replacement. Cancer 1978;42:2177-81.

28. Robinson BG, Hales IB, Henniker KHO, et al. The effect of o-p'DDD on adrenal steroid replacement therapy requirements. Clin Endocrinol 1987;27:437-44.

29. Bugg MF, Ribeiro RC, Roberson PK, et al. Correlation of pathologic features with clinical outcome in pediatric adrenocortical neoplasia. Am J Clin Pathol 1994;101:6259.

30. Patil KK, Ransley PG, McCullagh M, et al. Functioning adrenocortical neoplasms in children. BJU International 2002;89:562-5.

Endereço para correspondência:

Romolo Sandrini

Unidade de Endocrinologia Pediátrica

Departamento de Pediatria - Hospital de Clínicas

R. Padre Camargo 250

80060-240 - Curitiba - PR

e-mail: sandrini@ufpr.br 